\title{
A NOTION OF CAPACITY WHICH CHARACTERIZES REMOVABLE SINGULARITIES $\left({ }^{1}\right)$
}

\author{
B Y
}

\author{
REESE HARVEY AND JOHN C. POLKING
}

\begin{abstract}
In this paper the authors define a capacity for a given linear partial differential operator acting on a Banach space of distributions. This notion has as special cases Newtonian capacity, analytic capacity, and $A C$ capacity. It is shown that the sets of capacity zero are precisely those sets which are removable sets for the corresponding homogeneous equation. Simple properties of the capacity are derived and special cases examined.
\end{abstract}

Introduction. In a previous paper [9] the authors gave sufficient conditions in terms of the Hausdorff measure of a set $A$ that the set $A$ be removable for certain classes of solutions to a homogeneous linear differential equation (see Definition 1.3 below). There are various classical results which give necessary and sufficient conditions for a set to be removable. For example, if $A$ is a compact subset of $\mathbf{R}^{n}$, then $A$ is removable for $L^{\infty}\left(\mathbf{R}^{n}\right)$ and the Laplacian, $\Delta$, if and only if the capacity of $A$ is zero; or if $A$ is a compact subset of $\mathbf{C}$ then $A$ is removable for $L^{\infty}(\mathbf{C})$ and $\partial / \partial \bar{z}$ if and only if the analytic capacity of $A$ is zero. This paper is prompted by a desire to understand and generalize these two results. (In particular, we obtain a new proof of these classical results.)

In $\oint_{1}$ we introduce a notion of capacity for a differential operator $P(x, D)$ on a Banach space $B$. This notion of capacity can be used to characterize removable sets (Theorem 1.4) for a wide class of differential operators.

In $\$ 2$ we give an alternate "dual" definition of capacity for $L^{p}(1<p \leq \infty)$; and introduce a general concept of capacitary potential and capacitary mass. A uniqueness result for $1<p<\infty$ is obtained.

In $\$ 3$ we examine the $L^{\infty}$-capacities for the Laplacian, the Cauchy-Riemann operator, the heat operator, and the square of the Cauchy-Riemann operator. In particular, the classical notions of $L^{\infty}$-capacity for the Laplacian and the CauchyRiemann operator are seen to be special cases of the $L^{\infty}$-capacity introduced in Definition 1.2. Perhaps our notion of $L^{\infty}$-capacity for the heat operator will find

Received by the editors August 31, 1971.

AMS 1970 subject classifications. Primary 35099; Secondary 31C15.

Key words and phrases. Partial differential operators, capacity, removable singularities, capacitary potential, capacitary mass.

(1) This research was partially supported by NSF Grant 19011 and NSF Grant GP 23400 . 
application to a Wiener criterion for the Dirichlet problem for the heat equation.

At the time this work was done the authors were unaware of the related capacity studied by Fuglede ([6] and [7]), Meyers [11] and Reshetnyak [13]. While the intersection of these two concepts is large neither contains the other. The two can be compared only when the differential operator has a fundamental solution $E$ which is a kernel of the type considered in [11] and [13], i.e. $E$ must be positive and lower semicontinuous. If $E$ has these properties then the difference between the capacities is that in Definition 1.2 we consider potentials of distributions $u$ compactly supported in $A$, while Meyers and Reshetnyak only consider potentials of positive measures. There is an obvious inequality between the two capacities. An inequality in the opposite direction is sometimes true. For the Laplacian and the heat operator on $L^{\infty}$ this is shown in Theorems 3.1 and 3.2. For $(1-\Delta)^{S / 2}$ and $L^{p}$ see [1]. However, analytic capacity probably cannot be described in terms of measures.

The capacity of Meyers and Reshetnyak has nice set theoretic properties. In particular it is easily seen to be a capacity in the sense of Choquet. This is probably not possible for our capacity. (In particular, it is unknown in the case of analytic capacity.) On the other hand the capacity defined in this paper has application to problems in partial differential equations, as illustrated by Theorem 1.4, and the uniform approximation results of $[12]$ for elliptic equations.

1. Capacity and removable singularities. Suppose $P(x, D)$ is a linear partial differential operator with $C^{\infty}$ coefficients defined on an open set $\Omega \subset \mathbf{R}^{n}$. Let $B$ denote a Banach space contained in $\mathscr{T}^{\prime}(\Omega)$.

Definition 1.1. For each set $A \subset \Omega$, the $B$-capacity, denoted $B$-Cap $P(A, \Omega)$ of $A$ with respect to $P(x, D)$ is

$\sup \{|(P(x, D) f, 1)|: f \in B,\|f\| \leq 1$, and $P(x, D) f$ is compactly supported in $A\}$.

Suppose $P(D)$ has constant coefficients and $E \in \mathscr{D}^{\prime}\left(\mathbf{R}^{n}\right)$ is a fundamental solution for $P(D)$. That is, $P(D) E=\delta$. The next definition is the same as Definition 1.1 except that only functions $f$ of the form $E * u$ are considered.

Definition 1.2. For each set $A \subset \mathbf{R}^{n}$, the $B$-capacity, denoted $B-\operatorname{Cap}_{E}(A)$, of $A$ with respect to $E$ is

$$
\sup \left\{|(u, 1)|: u \in \mathcal{E}^{\prime}\left(\mathbf{R}^{n}\right), \operatorname{supp} u \subset A, E * u \in B \text {, and }\|E * u\| \leq 1\right\} .
$$

We will be particularly interested in capacities modeled on three types of Banach spaces: the spaces $L^{p}(\Omega)$ with $1 \leq p \leq \infty$, the Banach space $B C(\Omega)$ of bounded continuous functions on $\Omega$ with the uniform norm, and the spaces $\operatorname{Lip}^{\delta}(\Omega)$ with $0<\delta \leq 1$, consisting of continuous functions on $\Omega$ which satisfy a uniform Hölder condition of order $\delta$. The norm in $\operatorname{Lip}^{\delta}(\Omega)$ is defined by 


$$
\|f\|_{\operatorname{Lip}(\Omega)} \delta\|f\|_{\infty}+\sup _{x, y \in \Omega}\left(|f(x)-f(y)| /|x-y|^{\delta}\right) .
$$

In these special cases the capacity of $A$ with respect to $P(x, D)$ will be denoted by $L^{p}-\operatorname{Cap}_{P}(A, \Omega), B C-\operatorname{Cap}_{P}(A, \Omega)$, and $\operatorname{Lip}^{\delta}-\operatorname{Cap}_{P}(A, \Omega)$ respectively; and the capacity of $A$ with respect to $E$ will be denoted by $L^{p}-\operatorname{Cap}_{E}(A), B C-\operatorname{Cap}_{E}(A)$, and $\operatorname{Lip}^{\delta}-\operatorname{Cap}_{E}(A)$ respectively.

The following properties of capacity are obvious:

$$
B-\operatorname{Cap}_{P}(A, \Omega) \geq 0
$$

for all $A \subset \Omega$,

$B-\operatorname{Cap}_{P}\left(A_{1}, \Omega\right) \leq B-\operatorname{Cap}_{P}\left(A_{2}, \Omega\right)$ if $A_{1} \subset A_{2} \subset \Omega$,

$$
B-\operatorname{Cap}_{P}(A, \Omega)=\sup \left\{B-\operatorname{Cap}_{P}(K, \Omega): K \subset A \text { is compact }\right\} \text { for all } A \subset \Omega \text {. }
$$

The capacity $B$-Cap $E(A)$ also clearly has properties (1.1), (1.2), and (1.3).

Let $\mathcal{F}$ be a class of distributions in $\Omega$.

Definition 1.3. A relatively closed set $A \subset \Omega$ is removable for $\mathcal{F}$ with respect to $P(x, D)$ if each $f \in \mathcal{F}$ which satisfies $P(x, D) f=0$ in $\Omega \sim A$ also satisfies $P(x, D) f=0$ in $\Omega$.

If a set $A \subset \Omega$ is removable for $B$ with respect to $P(x, D)$ then obviously the $B$-capacity of $A$ with respect to $P(x, D)$ is zero. In the following theorem we establish the converse fact for a wide class of differential operators and special Banach spaces. Let $P^{(\alpha)}(\xi)$ denote $(\partial / \partial \xi)^{a} P(\xi)$ for a polynomial $P(\xi)$, and let $M_{\text {loc }}(\Omega)$ denote the space of regular Borel measures on $\Omega$.

Theorem 1.4. Suppose $P(D)$ bas a fundamental solution $E$ with $P^{(a)}(D) E \in$ $\mathbb{M}_{\text {loc }}\left(\mathbf{R}^{n}\right)$ for each $a$. Let $A$ be a relatively closed subset of $\mathbf{\Omega} \subset \subset \mathbf{R}^{n}$.

(a) The set $A$ is removable for $L_{10 \mathrm{lo}}^{p}(\Omega), 1<p \leq \infty$, if and only if $L^{p}-\operatorname{Cap}_{P}(A, \Omega)=0$.

(b) The set $A$ is removable for $C(\Omega)$ if and only if $B C-\operatorname{Cap}_{P}(A, \Omega)=0$.

(c) The set $A$ is removable for $\operatorname{Lip}_{1 \text { oc }}^{\delta}(\Omega), 0<\delta \leq 1$, iff $\operatorname{Lip}^{\delta}-\operatorname{Cap}_{P}(A, \Omega)=0$.

Remark 1. The hypotheses of Theorem 1.4 are satisfied by elliptic and parabolic operators. More generally semielliptic (see [10, p. 102]) operators satisfy the hypotheses. Moreover, the applicability of Theorem 1.4 is not limited to hypoelliptic operators. For example, the wave operator $\partial^{2} / \partial t^{2}-\partial^{2} / \partial x^{2}$ in $\mathbf{R}^{2}$ satisfies the hypotheses of Theorem 1.4.

Remark 2. Suppose $P(x, D)$ is an elliptic differential operator $w$ ith infinitely differentiable coefficients in $\Omega$ which has a fundamental solution. (For example this is always the case if the coefficients are real-analytic.) Then the analog of Theorem 1.4 is true for $P(x, D)$. The proof is essentially the same.

Proof. Let $B$ denote one of the above Banach spaces and suppose that 
$B$-Cap $P(A, \Omega)=0$. Assume that $f \in B$ satisfies $P(D) f=0$ in $\Omega-A$. Given $\phi \in C_{0}^{\infty}(\Omega)$, let $g=E *(\phi P(D) f)$. Then $P(D) g=\phi P(D) f$. It suffices to show that $g \in B$ because the hypothesis $B-\operatorname{Cap}_{P}(A, \Omega)=0$ would then imply that $(P(D) f, \phi)=(\phi P(D) f, 1)=(P(D) g, 1)=0$.

Let $\psi \in C_{0}^{\infty}(\Omega)$. Then by the generalized Leibniz formula we have

$$
\begin{aligned}
& (\phi P(D) f, \psi)=\left(f,{ }^{t} P(D)(\phi \psi)\right) \\
& \quad=\sum\left((-1)^{|\alpha|} / \alpha !\right)\left(f, D^{\alpha} \phi^{t} P^{(\alpha)}(D) \psi\right)=\left(\sum\left((-1)^{|\alpha|} / \alpha !\right) P^{(\alpha)}(D)\left(f D^{\alpha} \phi\right), \psi\right) .
\end{aligned}
$$

Consequently we have the adjoint Leibniz formula

$$
\phi P(D) f=\sum_{|\alpha| \geq 0} \frac{(-1)^{|\alpha|}}{\alpha !} P^{(\alpha)}(D)\left(f D^{a} \phi\right)
$$

Therefore

$$
g=E *(\phi P(D) f)=\sum_{|a| \geq 0} \frac{(-1)^{|a|}}{a !}\left(P^{(\alpha)}(D) E\right) *\left(f D^{a} \phi\right) .
$$

For each of the Banach spaces $B$ under consideration convolution by $P^{(\alpha)}(D) E$, followed by restriction to $\Omega$, is a continuous linear mapping of $B$ into $B$, since $P^{(\alpha)}(D) E \in \mathbb{M}_{\text {loc }}\left(\mathbf{R}^{n}\right)$ and $\Omega \subset \subset \mathbf{R}^{n}$. Consequently (since each $f D^{\alpha} \phi \in B$ ) the formula (1.5) implies that $g \in B$.

Under additional assumptions concerning the behavior of $P^{(a)}(D) E$ near infinity, Theorem 1.4 can be improved considerably. Let $\psi \in C^{\infty}\left(\mathbf{R}^{n}\right)$ denote a function which vanishes in a neighborhood of the origin and is identically one in a neighborhood of infinity.

Theorem 1.5. Suppose $P(D)$ bas a fundamental solution $E$ with $P^{(a)}(D) E \epsilon$ $\pi_{\text {loc }}\left(\mathbf{R}^{n}\right)$ for all $\alpha$. Let $A$ be a relatively closed subset of an arbitrary open set $\Omega \subset \mathbf{R}^{n}$.

(a) $(1<p \leq \infty)$ Suppose $\psi P^{(\alpha)}(D) E \in L^{p}\left(\mathbf{R}^{n}\right)$ for all $\alpha$. The set $A$ is removable for $L_{1 \text { oc }}^{p}(\Omega)$ if and only if $L^{p}-\operatorname{Cap}_{E}(A)=0$.

(b) Suppose $\psi P^{(a)}(D) E \in L^{\infty}$ for all a. The set $A$ is removable for $C(\Omega)$ if and only if $B C-\mathrm{CaP}_{E}(A)=0$.

Proof. We assume the notation in the proof of Theorem 1.4. The proof of Theorem 1.5 is the same except that one must verify that each $\left(P^{(\alpha)}(D) E\right)^{*}$ $\left(f D^{\alpha} \phi\right)$ belongs to $L^{p}\left(\mathbf{R}^{n}\right)$ or $B C\left(\mathbf{R}^{n}\right)$. By hypothesis $(1-\psi) P^{(\alpha)}(D) E$ is a compactly supported regular Borel measure while $f D^{a} \phi \in L^{p}\left(\mathbf{R}^{n}\right)$. Therefore $\left((1-\psi) P^{(\alpha)}(D) E\right) * f D \phi \in L^{p}\left(\mathbf{R}^{n}\right)$. By hypothesis, $\psi P^{(a)}(D) E \in L^{p}\left(\mathbf{R}^{n}\right)$, while $f D^{\alpha} \phi \in L^{1}\left(\mathbf{R}^{n}\right)$. Therefore $\left(\psi P^{(\alpha)}(D) E\right) * f D^{a} \phi \in L^{p}\left(\mathbf{R}^{n}\right)$.

To prove part (b) of the theorem note that $f D^{\alpha} \phi$ is a compactly supported 
continuous function. Since $(1-\psi) P^{(a)}(D) E$ is compactly supported measure and $\psi P^{(\alpha)}(D) E \in L^{\infty}\left(\mathbf{R}^{n}\right)$, this implies that $\left(P^{(\alpha)}(D) E\right) *\left(f D^{\alpha} \phi\right) \in B C\left(\mathbf{R}^{n}\right)$.

The proof of Theorem 1.4 and Theorem 1.5 can be used to gain additional information about capacities. For example, if the capacity of a set is locally zero then it is globally zero. More precisely, under the hypothesis of Theorem 1.5 we have

Corollary 1.6. If $L^{p}-\operatorname{Cap}_{E}(A) \neq 0$ then there exists a point $x \in A$ such that $L^{p}-\mathrm{Cap}_{E}(A \cap K) \neq 0$ for every compact neighborbood $K$ of $x$.

Similar results corresponding to Theorem 1.4 instead of Theorem 1.5 follow just as easily as Corollary 1.6.

Remark 1. Let $B$ denote one of the three Banach spaces $L^{p}(\Omega)(1<p \leq \infty)$, $B C(\Omega)$ or $\operatorname{Lip}^{\delta}(\Omega)$. Assume the hypotheses of Theorem 1.5 are satisfied. It follows from the proof of Theorem 1.5 that $B$-Cap $P(A, \Omega)=0$ if and only if $B-\operatorname{Cap}_{E}(A)=0$.

Remark 2. If $\Omega \subset \subset \mathbf{R}^{n}$ and $P(D)$ and $Q(D)$ belong to the same semielliptic class (for example if $P(D)$ and $Q(D)$ are both elliptic of order $m$ ) then $L^{p}-\operatorname{Cap}_{P}(A, \Omega)=0$ if and only if $L^{p}-\operatorname{Cap}_{Q}(A, \Omega)=0$ for $1<p<\infty$. This fact follows easily from the Fabes-Riviere inequalities [5] (the Calderón-Zygmund inequalities if $P(D)$ and $Q(D)$ are elliptic).

Remark 3. If $1<p<\infty$ and the Hausdorff measure of $A$ in dimension $n-m q$ is locally finite then $L^{p}$-Cap $P(A, \Omega)=0$ for all operators $P(x, D)$ of order $m$. This follows easily from Lemma 3.2 in [9] and Theorem 2.1 in the next section.

Remark 4. If $P(x, D)$ is elliptic of order $m$ and has a fundamental solution, then $\operatorname{Lip}^{\delta}-\operatorname{Cap}_{P}(A, \Omega)=0$ if and only if $\Lambda_{n-m}+\delta(A)=0$ (see [4, p. 91]) and Theorem 4.4 in [9]; $\Lambda_{k}$ denotes $k$ dimensional Hausdorff measure).

2. Capacitary extremals. The results of this section are similar to results in Fuglede ([6], [7]), Meyers [11], and Reshetnyak [13]; however the proofs are some what easier.

The next result gives an alternate definition for the $L^{p}$-capacity of a compact set which is important in applications. Here $q$ is defined by $1 / q+1 / p=1$. No assumptions on the operator $P(x, D)$ are required other than smooth coefficients.

Theorem 2.1. $(1<p<\infty)$ Let $K$ denote a compact subset of $\Omega$.

$$
L^{p}-\operatorname{Cap}_{P}(K, \Omega)=\inf \left\{\left\|^{t} P(x, D) g\right\|_{q}: g \in C_{0}^{\infty}(\Omega) \text { and } g \equiv 1 \text { in a nbd of } K\right\} \text {. }
$$

Proof. Let $c$ denote the infimum defined above. First suppose $f \in L^{p}(\Omega)$, $\|f\|_{p} \leq 1$ and $\operatorname{supp} P(x, D) f \subset K$; and that $g \in C_{0}^{\infty}(\Omega)$ satisfies $g \equiv 1$ in a neighborhood of $K$. Then by Hölder's inequality 


$$
|(P(x, D) f, 1)|=|(P(x, D) f, g)|=\left|\left(f,{ }^{t} P(x, D) g\right)\right| \leq\left\|{ }^{t} P(x, D) g\right\|_{q} .
$$

Consequently $L^{p}$-Cap $P(K, \Omega) \leq c$.

To prove the converse, pick $b \in C_{0}^{\infty}(\Omega)$ with $b \equiv 1$ in a neighborhood of $K$. Then $c$ is the distance in $L^{q}(\Omega)$ from the point ${ }^{t} P(x, D) b$ to the subspace ${ }^{t} P(x, D) C_{0}^{\infty}(\Omega \sim K)$. By the Hahn-Banach theorem there is a linear functional $f \epsilon$ $L^{p}(\Omega)$ with $\|f\|_{p}=1$ such that $f$ annihilates the subspace ${ }^{t} P(x, D) C_{0}^{\infty}(\Omega \sim K)$ and $\left(f,{ }^{t} P(x, D) b\right)$ is the distance in $L^{q}(\Omega)$ from the point ${ }^{t} P(x, D) b$ to the subspace ${ }^{t} P(x, D) C_{0}^{\infty}(\Omega \sim K)$. Since $\left(f,{ }^{t} P(x, D) \phi\right)=0$ for all $\phi \in C_{0}^{\infty}(\Omega \sim K)$ we have $P(x, D) f=0$ in $\Omega \sim K$. Consequently,

$$
c=\left(f,{ }^{t} P(x, D) b\right)=(P(x, D) f, b)=(P(x, D) f, 1) \leq L^{p}-\operatorname{Cap}_{P}(K, \Omega) .
$$

Remark. The proof shows that the norm of the linear functional ${ }^{t} P(x, D) b$ acting on the subspace $N=\left\{f \in L^{p}(\Omega): P(x, D) f=0\right.$ in $\left.\Omega \sim K\right\}$ of $L^{p}(\Omega)$ is the same as the norm of ${ }^{t} P(x, D) b$ in the quotient space, $L^{q}(\Omega)$ modulo the closure of ${ }^{t} P(x, D) C_{0}^{\infty}(\Omega \sim K)$. Note that the closure of ${ }^{t} P(x, D) C_{0}^{\infty}(\Omega \sim K)$ in $L^{q}(\Omega)$ is the annihilator of $N$.

Theorem 2.2. $(1<p<\infty)$ Let $K$ denote a compact subset of $\mathbf{\Omega}$.

(a) There is a unique function $f \in L^{p}(\Omega)$ with $\|f\|_{p} \leq 1$ and $P(x, D) f=0$ in $\mathbf{\Omega} \sim K$ such that $(P(x, D) f, 1)=L^{p}-\operatorname{Cap}_{P}(K, \Omega)$.

(b) There is a unique function $v \in L^{q}(\Omega)$ belonging to the closure of $\left\{{ }^{t} P(x, D) g: g \in C_{0}^{\infty}(\Omega)\right.$ and $g \equiv 1$ in a nbd of $\left.K\right\}$ such that $\|v\|_{q}=L^{p}-\mathrm{Cap}_{P}(K, \Omega)$.

(c) The extremals in (a) and (b) are related by

$$
v=L^{p}-\operatorname{Cap}_{P}(K, \Omega)(\bar{f} /|f|)|f|^{p / q}, \quad f=\left(L^{p}-\operatorname{Cap}_{P}(K, \Omega)\right)^{-q / p}(\bar{v} /|v|)|v|^{q / p}
$$

and the product $f \cdot v$ is a pos itive integrable function on $\Omega$ with $\int_{\Omega} f \cdot v d x=$ $L^{p}-\operatorname{Cap}_{P}(K, \Omega)$.

Proof. Let $b \in C_{0}^{\infty}(\Omega)$ with $b \equiv 1$ in a neighborhood of $K$ and let $F=\{f \epsilon$ $L^{p}(\Omega):\|f\|_{p} \leq 1$ and $P(x, D) f=0$ in $\left.\Omega \sim K\right\}$. Then $L^{p}-\operatorname{Cap}_{P}(K, \Omega)=$ $\sup _{f \in F}\left|\left(f,{ }^{t} P b\right)\right|$. Since by Alaoglu's theorem $F$ is weakly compact, this supremum is attained at a point $f \in F$, which provides an extremal $f$ for part (a). Since the set $\left\{{ }^{t} P(x, D) g: g \in C_{0}^{\infty}(\Omega), g \equiv 1\right.$ in a neighborhood of $K$, and $\left\|{ }^{t} P(x, D) g\right\|_{q} \leq$ $2 L^{p}$ - $\left.\operatorname{Cap}_{P}(K, \Omega)\right\}$ is weakly relatively compact in $L^{q}(\Omega)$ there exists an extremal $v$ for part (b).

To prove part (c) let $f$ and $v$ be any such extremals. Since $(P(x, D) f, 1)=$ $\left(f,{ }^{t} P(x, D) g\right)$ for all $g \in C_{0}^{\infty}(\Omega)$ with $g \equiv 1$ near $K$, we have the important fact that 


$$
(P(x, D) f, 1)=(f, v) .
$$

Consequently,

$$
L^{p}-\operatorname{Cap}_{P}(K, \Omega)=(P(x, D) f, 1)=(f, v) \leq\|f\|_{p}\|v\|_{q} \leq L^{p}-\operatorname{Cap}_{P}(K, \Omega) .
$$

Therefore we must have equality throughout and in particular we must have equality for Hölder's inequality. This proves that $f$ and $v$ satisfy the relations of part (c) and are therefore uniquely determined.

The unique extremal function $f$ in part (a) of Theorem 2.2 will be called the $L^{p}$-capacitary function for $K$ with respect to $P(x, D)$ and $\Omega$. The unique extremal $v$ in part (b) of Theorem 2.2 will be called the $L^{p}$-capacitary mass for $K$ with respect to $P(x, D)$ and $\Omega$.

Next we investigate the analogs of Theorem 2.1 and 2.2 for $p=\infty$. Another capacity arises in this case. Let $C_{\infty}(\Omega)$ denote the space of continuous functions in $\Omega$ which vanish at infinity and at the boundary of $\Omega$. Consistent with the notation of $\$ 1$, let

$$
\left.C_{\infty}-\operatorname{Cap}_{P}(A, \Omega)=\sup \{\mid P(x, D) f, 1) \mid: f \in C_{\infty}(\Omega),\|f\|_{\infty} \leq 1 \text { and } P(x, D) f=0 \text { in } \Omega \sim K\right\} .
$$

Since $C_{\infty}(\Omega)$ is a closed subspace of $L^{\infty}(\Omega), C_{\infty}-\operatorname{Cap}_{P}(K, \Omega) \leq L^{\infty}-\operatorname{Cap}_{P}(K, \Omega)$. The dual space $C_{\infty}(\Omega)^{\prime}$ consists of all regular Borel measures with finite total variation. For $v \in C_{\infty}(\Omega)^{\prime}$ the norm of $v$ is the total variation of $v$. With this topology $L^{1}(\Omega)$ is a closed subspace of $C_{\infty}(\Omega)^{\prime}$.

Theorem 2.3. Let $K$ denote a compact subset of $\Omega$.

(a) There exists a function $f \in L^{\infty}(\Omega)$ with $\|f\|_{\infty} \leq 1$ and $P(x, D) f=0$ in $\Omega \sim K$ such that $(P(x, D) f, 1)=L^{\infty}-\operatorname{Cap}_{P}(K, \Omega)$.

(b) $L^{\infty}-\operatorname{Cap}_{P}(K, \Omega)=\inf \left\{\left\|{ }^{t} P(x, D) g\right\|_{1}: g \in C_{0}^{\infty}(\Omega)\right.$ and $g \equiv 1$ in a neigbborbood of $\left.K\right\}$.

(c) There exists a measure $v \in C_{\infty}(\Omega)^{\prime}$ belonging to the weak, closure of $\left\{{ }^{t} P(x, D) g: g \in C_{0}^{\infty}(\Omega)\right.$ and $g \equiv 1$ in a neighborbood of $\left.K\right\}$ sucb that $\|v\|=$ $C_{\infty}-\operatorname{Cap}_{P}(K, \Omega)$.

Remark. Let $R$ denote the closure of ${ }^{t} P(x, D) C_{0}^{\infty}(\Omega \sim K)$ in $L^{1}(\Omega)$ and let $\bar{R}$ denote the weak ${ }^{*}$ closure of the same subspace in $C_{\infty}(\Omega)^{\prime}$. Pick $b \in C_{0}^{\infty}(\Omega)$ with $b \equiv 1$ in a neighborhood of $K$. Part (b) of Theorem 2.3 can be interpreted as saying that $L^{\infty}$-Cap $(K, \Omega)$ is equal to the distance in $L^{1}(\Omega)$ from ${ }^{t} P(x, D) b$ to $R$. Part (c) on the other hand says that $C_{\infty}-\operatorname{Cap}_{P}(K, \Omega)$ is equal to the distance in $C_{\infty}(\Omega)^{\prime}$ from ${ }^{t} P(x, D) b$ to $\bar{R}$.

Proof. The proof of part (a) is the same as the proof of Theorem 2.2(a). The 
proof of part (b) is the same as the proof of Theorem 2.1. To prove part (c), notice that the set $F=\left\{{ }^{t} P(x, D) g: g \in C_{0}^{\infty}(\Omega), g \equiv 1\right.$ in a neighborhood of $K$, and $\left\|{ }^{t} P(x, D) g\right\|_{1} \leq 2 L^{\infty}$-Cap $\left.P(K, \Omega)\right\}$ is weak ${ }^{*}$ relatively compact in $C_{\infty}(\Omega)^{\prime}$. Consequently there is a net $\left\{g_{a}\right\} \subset F$ which converges in the weak ${ }^{*}$ topology to a measure $v \in C_{\infty}(\Omega)^{\prime}$ which has the property that $\|v\|$ is the distance from ${ }^{t} P(x, D) b$ to $\bar{R}$. Here we use the notation of the above remark. The continuous capacity $C_{\infty}-\operatorname{Cap}_{P}(K, \Omega)$ is bounded above by $\|v\|$ since if $f \in C_{\infty}(\Omega)$ satisfies $\|f\|_{\infty} \leq 1$ and $P(x, D) f=0$ in $\Omega \sim K$, then $\|(P(x, D) f, 1)|=|\left(f,{ }^{t} P(x, D) g_{\alpha}\right)|=|(f, v) \mid \leq$ $\|f\|_{\infty}\|v\| \leq\|v\|$. That $\|v\| \leq C_{\infty}-\operatorname{Cap}_{P}(K, \Omega)$ can be seen as follows. For each $\epsilon>0$, there is a function $f_{\epsilon} \in C^{\infty}(\Omega)$ with $\left\|f_{\epsilon}\right\|_{\infty} \leq 1$ such that $f_{\epsilon}$ annihilates ${ }^{t} P(x, D) C_{0}^{\infty}(\Omega \sim K)$ and $\left(f_{\epsilon}, P(x, D) b\right)+\epsilon$ is greater than the distance from ${ }^{t} P(x, D) b$ to $\bar{R}$. Hence $\|v\| \leq\left(f_{\epsilon},{ }^{t} P(x, D) b\right)+\epsilon=\left(P(x, D) f_{\epsilon}, b\right)+\epsilon$. Since $f_{\epsilon}$ annihilates ${ }^{t} P(x, D) C_{0}^{\infty}(\Omega \sim K), P(x, D) f_{\epsilon}=0$ in $\Omega \sim K$. Consequently $\left(P(x, D) f_{\epsilon}, b\right)=\left(P(x, D) f_{\epsilon}, 1\right)$ and $\|v\| \leq C_{\infty}-\operatorname{Cap}_{P}(K, \Omega)+\epsilon$ for all $\epsilon>0$. Hence $\|v\|=C_{\infty}-\operatorname{Cap}_{P}(K, \Omega)$.

The previous results of this section have analogs for the capacities $L^{p}-\mathrm{Cap}_{E}(K)$.

Theorem 2.4. $(1<p<\infty)$ Let $K$ denote a compact subset of $\mathbf{R}^{n}$.

$$
L^{p}-\operatorname{Cap}_{E}(K)=\inf \left\{\|\psi\|_{q}: \psi \in C_{0}^{\infty}\left(\mathbf{R}^{n}\right) \text { and } \check{E} * \psi \equiv 1 \text { in a neighborbood of } K\right\} \text {. }
$$

Proof. The proof is a modification of the proof of Theorem 2.1. Let $c$ denote the infimum defined above. First, if $E * u \in L^{p}\left(\mathbf{R}^{n}\right),\|E * u\|_{p} \leq 1, u \in \mathcal{E}_{K}^{\prime}\left(\mathbf{R}^{n}\right)$, and $\psi \in C_{0}^{\infty}\left(\mathbf{R}^{n}\right)$, with $\check{E} * \psi \equiv 1$ in a neighborhood of $K$, then $|u(1)|=$ $|(u, \check{E} * \psi)|=|(E * u, \psi)| \leq\|\psi\|_{q}$, by Hölder's inequality. Hence $L^{p}-$ Cap $_{E}(K) \leq c$.

To prove the converse, pick $b \in C_{0}^{\infty}\left(\mathbf{R}^{n}\right)$ with $b \equiv 1$ in a neighborhood of $K$. Let $R$ denote the closure in $L^{q}\left(\mathbf{R}^{n}\right)$ of the set of $\phi \in C_{0}^{\infty}\left(\mathbf{R}^{n}\right)$ such that $\check{E} * \phi \equiv$ 0 in a neighborhood of $K$. Then $c$ is the distance in $L^{q}\left(\mathbf{R}^{n}\right)$ from the point ${ }^{t} P(D) b$ to the subspace $R$. By the Hahn-Banach theorem there exists a linear functional $f \in L^{p}\left(\mathbf{R}^{n}\right)$ with $\|f\|_{p}=1$ such that $f$ annihilates $R$ and $\left(f,{ }^{t} P(D) b\right)$ is the distance in $L^{q}\left(\mathbf{R}^{n}\right)$ from the point ${ }^{t} P(D) b$ to the subspace $\mathbf{R}$. Since $f$ annihilates ${ }^{t} P(D) C_{0}^{\infty}\left(\mathbf{R}^{n} \sim K\right)$, we have $P(D) f=0$ in $\mathbf{R}^{n} \sim K$.

Let $u=P(D) f$. Since $(E * u, \phi)=(u, \breve{E} * \phi)$ for all $\phi \in C_{0}^{\infty}\left(\mathbf{R}^{n}\right)$, the distribution $E * u$, and hence $f-E * u$, annihilates $R$. Also $P(f-E * u)=0$ in $\mathbf{R}^{n}$. Given $g \in C_{0}^{\infty}\left(\mathbf{R}^{n}\right)$, let $\phi=b \breve{E} * g$ and $\psi=g-{ }^{t} P \phi$. Then $g={ }^{t} P \phi+\psi$ with $\phi \in C_{0}^{\infty}\left(\mathbf{R}^{n}\right)$ and $\check{E} * \psi=0$ in a neighborhood of $K$. Consequently, $(f-E * u, g)=$ $\left(f-E * u,{ }^{t} P \phi\right)+(f-E * u, \psi)=0$. Therefore $f=E * u$ and hence $c=$ $\left(f,{ }^{t} P(D) b\right)=(P(D) f, b)=(u, 1) \leq L^{p}-$ Cap $_{E}(K)$.

Theorem 2.5. $(1<p<\infty)$ Let $K$ denote a compact subset of $\mathbf{R}^{n}$. 
(a) There is a unique function $f=E * u \in L^{p}\left(\mathbf{R}^{n}\right)$ with $\|E * u\|_{p} \leq 1$ and $u \in \mathcal{E}_{K}^{\prime}\left(\mathbf{R}^{n}\right)$ such that $(u, 1)=L^{p}-\mathrm{Cap}_{E}(K)$.

(b) There is a unique function $v \in L^{q}\left(\mathbf{R}^{n}\right)$ belonging to the closure of the set of $\psi \in C_{0}^{\infty}\left(\mathbf{R}^{n}\right)$ with $\check{E} * \psi \equiv 1$ in a neigbborbood of $K$ such that $\|v\|_{q}=$ $L^{p}-\mathrm{Cap}_{E}(K)$.

(c) The extremals in (a) and (b) are related by

$$
v=L^{p}-\operatorname{Cap}_{E}(K)(f /|f|)|f|^{p / q}, \quad f \doteq\left(L^{p}-\operatorname{CaP}_{E}(K)\right)^{-q / p}(\bar{v} /|\nu|)|v|^{q / p}
$$

and the product $f \cdot v$ is a positive integrable function on $\mathbf{R}^{n}$ with $\int_{\mathbf{R}^{n}} f \cdot v=$ $L^{p}-\mathrm{CaP}_{E}(K)$.

The proof is the same as the proof of Theorem 22 . The extremals $f$ and $v$ will be referred to as the $L^{p}$-capacitary potential and the $L^{p}$-capacitary mass, respectively, with respect to $E$.

The analog of Theorem 2.3 is

Theorem 2.6. Let $K$ denote a compact subset of $\mathbf{R}^{n}$.

(a) There exists a function $f=E * u \in L^{\infty}\left(\mathbf{R}^{n}\right)$ with $\|f\|_{\infty} \leq 1$ and $u \epsilon$ $\xi_{K}^{\prime}\left(\mathbf{R}^{n}\right)$ such that $(P(D) f, 1)=L^{\infty}-\operatorname{Cap}_{E}(K)$.

(b) $L^{\infty}-\operatorname{Cap}_{E}(K)=\inf \left\{\|\psi\|_{1}: \psi \in C_{0}^{\infty}\left(\mathbf{R}^{n}\right)\right.$ and $\check{E} * \psi \equiv 1$ in a neighborbood of $\left.K\right\}$.

(c) There exists a measure $v \in C_{\infty}\left(\mathbf{R}^{n}\right)^{\prime}$ belonging to the weak ${ }^{*}$ closure of $\left\{\psi \in C_{0}^{\infty}\left(\mathbf{R}^{n}\right): E * \psi \equiv 1\right.$ in a neighborbood of $\left.K\right\}$ such that $\|v\|=C_{\infty}-\mathrm{Cap}_{E}(K)$.

The proof is essentially the same as the proof of Theorem 2.3.

Notice that $p=\infty$ is allowed in part (b) below.

Proposition 2.7. Suppose $K_{1} \supset K_{2} \supset \cdots$ is a sequence of compact sets and $K=\bigcap_{j=1}^{\infty} K_{j}$

(a) $(1<p<\infty)$ Let $f_{j}$ be the $L^{p}$-capacitary potential of $K_{j}$ with respect to $E$. Then the sequence $\left\{f_{j}\right\}$ converges weakly in $L^{p}\left(\mathbf{R}^{n}\right)$ to $f$, the $L^{p}$-capacitary potential of $K$ with respect to $E$.

(b) $(1<p \leq \infty) L^{p}-\operatorname{Cap}_{E}(K)=\lim _{j \rightarrow \infty} L^{p}-\operatorname{Cap}_{E}\left(K_{j}\right)$.

Remark. The same theorem holds with $L^{p}$-Cape replaced by $L^{p}$-Cap ${ }_{P}$.

Proof. Since the sequence $\left\{f_{j}\right\}$ is bounded in $L^{p}\left(R^{n}\right)$, it has a weakly convergent subsequence. It suffices to show that every weakly convergent subsequence $\left\{f_{j_{k}}\right\}$ converges to $f$. Let $g$ be the weak limit of $\left\{f_{j_{k}}\right\}$. Then $g$ is a potential, $P(D) g=0$ in $\mathbf{R}^{n} \sim K$ and $\|g\|_{p} \leq 1$. Consequently,

$$
L^{p}-\operatorname{Cap}_{E}(K) \geq(P(D) g, 1)=\lim _{k \rightarrow \infty}\left(P(D) f_{j_{k}}, 1\right)=\lim _{k \rightarrow \infty} L^{p}-\operatorname{CaP}_{E}\left(K_{j_{k}}\right) .
$$

The reverse inequality is true by $(1.2)$, so we have $L^{p}$-Cap $E(K)=(P(D) g, 1)$. 
Since the capacitary potential for $K$ is unique we have $g=f$. This proves all but part (b) with $p=\infty$. By the above reasoning the sequence $\left\{f_{j}\right\}$ has a convergent subsequence $\left\{f_{j_{k}}\right\}$, with $\lim _{k \rightarrow \infty} L^{\infty}-\operatorname{Cap}_{E}\left(K_{j_{k}}\right)=L^{\infty}-\operatorname{Cap}_{E}(K)$. Since $L^{\infty}$-Cap $E_{E}\left(K_{j}\right)$ is a nonincreasing sequence this proves that $L^{\infty}$-Cap $E(K)=$ $\lim _{j \rightarrow \infty} L^{\infty}-\operatorname{Cap}_{E}\left(K_{j}\right)$.

3. Examples. For $d / d x$ in $\mathbf{R}$ the analysis is extremely easy. A fundamental solution is the Heaviside function $Y(x)$ (the characteristic function of the positive real axis). If $K \subset \mathbf{R}$ is compact and nonempty, then $L^{\infty}$-Cap $Y(K)=1$. One can easily check that the $L^{\infty}$ capacitary potential for $K$ is uniquely determined on the unbounded components of $\mathbf{R}-K$. In fact, it is equal to 0 on the negative unbounded component and equal to 1 on the positive unbounded component.

The results for $\bar{\partial}=\partial / \partial \bar{z}$ are similar, although the verifications for $\partial / \partial \bar{z}$ are not all trivial. Let $E(z)=1 / \pi z$ denote the usual fundamental solution for $\partial / \partial \bar{z}$, and let $K$ denote a compact subset of C. If $f \in L^{\infty}(\mathbf{C})$ and $\partial f / \partial \bar{z}=u \in \mathcal{G}_{K}^{\prime}(\mathbf{C})$, then $f$ has a holomorphic extension across $\infty$, and $f=E * u$ if and only if $f(\infty)=$ 0 , by Liouville's theorem. Let $\Omega$ denote a bounded open set containing $K$ with $\partial \Omega$ smooth. Then

$$
\left(\frac{\partial}{\partial \bar{z}} f, 1\right)=\left(\frac{\partial}{\partial \bar{z}} f, \chi_{\Omega}\right)=\frac{1}{2 i} \int_{\partial \Omega} f(z) d z=\pi f^{\prime}(\infty) .
$$

The integration by parts is valid since the singular supports of $f$ and $\chi_{\Omega}$ are disjoint; and the last equality follows from the change of variables $w=1 / z$. If $\|f\|_{\infty} \leq 1$, let

$$
g(z)=(f(\infty)-f(z)) /(1-\overline{f(\infty)} f(z)) .
$$

Then $g(\infty)=0,\|g\|_{\infty} \leq 1$ and $\left|g^{\prime}(\infty)\right|=\left|f^{\prime}(\infty)\right| /\left(1-|f(\infty)|^{2}\right) \geq\left|f^{\prime}(\infty)\right|$. Consequently, $L^{\infty}$-Cap $\bar{\partial}(K)=L^{\infty}-\operatorname{Cap}_{E}(K)$. The analytic capacity $L^{\infty}-\operatorname{Cap}_{E}(K)$ was introduced by Ahlfors [2] and developed further by Garabedian [8].

If the complement of $K$ in the Riemann sphere is simply connected then, as is well known, the extremal problem

$$
\sup \left\{\left|f^{\prime}(\infty)\right|: f \in L^{\infty}(\mathrm{C}), \partial f / \partial \bar{z}=0 \text { in } \mathrm{C} \sim K, f(\infty)=0 \text {, and }\|f\|_{\infty} \leq 1\right\}
$$

has as unique solution the Riemann map of the complement of $K$ onto the unit disk with $f(\infty)=0$ and $f^{\prime}(\infty)>0$. Hence the $L^{\infty}$-capacitary potential is the Riemann map in this case. In general, the $L^{\infty}$-capacitary potential is unique in the unbounded component of $\mathrm{C} \sim K$ (see [4] for a proof).

Let $E(x)=-1 / \sigma_{n-1}(n-2)|x|^{n-2}$ denote the usual fundamental solution for the Laplacian $\Delta$ in $\mathbf{R}^{n}(n \geq 3)$. Let $K$ denote a compact subset of $\mathbf{R}^{n}$. Let $F$ denote the generalized solution to the Dirichlet problem for $\mathbf{R}^{n} \sim K$ with boundary 
values equal to one on $\partial K$. That is, $F$ is the balayage of the function 1 on $\mathbf{R}^{n}$ relative to $K$. Then $F$ is a superharmonic function on $\mathbf{R}^{n}$, i.e. $-\Delta F$ is a positive measure. Also $\Delta F=0$ in $\mathbf{R}^{n} \sim K, f=E * \Delta F$, and $\|F\|_{\infty}=1$.

Theorem 3.1. (a) The $L^{\infty}$-capacitary potential is unique in the unbounded component of $\mathbf{R}^{n} \sim K$ and coincides there with $-F$.

(b) $L^{\infty}-\operatorname{Cap}_{E}(K)=\sup \left\{\mu(1): \mu\right.$ is a positive measure on $K$ and $\left.\|E * \mu\|_{\infty} \leq 1\right\}$.

The constant $\left(\sigma_{n-1}(n-2)\right)^{-1}$ times the right-hand side of the above equation is usually taken as the definition of Newtonian capacity.

Proof. Let $B=B(0, R)$ be a ball which contains $K$ in its interior and let $\mu=-(n-2)_{\sigma} / R$ where $\sigma$ is the induced Lebesgue measure on $\partial B$. Then $E * \mu \equiv$ 1 in $B^{\circ}$. Let $f$ be any $L_{\infty}$-capacitary potential for $K$. Since $|f(x)| \leq 1, f$ vanishes at $\infty$ and $f$ is harmonic in $\mathbf{R}^{n} \sim K$, the maximum principle implies that $|f(x)| \leq F(x)$ in $\mathbf{R}^{n} \sim K$. Then $F+f \geq 0$ in $\mathbf{R}^{n} \sim K$ so

$$
\begin{aligned}
(\Delta(F+f), 1) & =(\Delta(F+f), \mu * E) \\
& =(E * \Delta(F+f), \mu)=(F+f, \mu) \leq 0 .
\end{aligned}
$$

Consequently, $(-\Delta F, 1) \geq(\Delta f, 1)$ so $-F$ is an $L^{\infty}$-capacitary potential. Then by (3.2) we have $(F+f, \mu)=0$. Since $F+f \geq 0$ and $\mu$ is a strictly negative measure, we must have $F+f \equiv 0$ on $\partial B$ and hence by the maximum principle $f=$ $-F$ in the unbounded component of $\mathbf{R}^{n} \sim K$.

Remark. Now using part (b) of Theorem 3.1 it follows as in [4, p. 15] that $C_{\infty}-\operatorname{Cap}_{E_{n}}(K)=L^{\infty}-\operatorname{Cap}_{E_{n}}(K)$ for all compact sets $K \subset \mathbf{R}^{n}$.

For the heat operator $H=\partial / \partial t-\Delta$ in $\mathbf{R}^{n+1}$ the results are similar to those for the Laplacian. The fundamental solution for $H$ is

$$
E(x, t)=(4 \pi t)^{-n / 2} Y(t) \exp \left(-|x|^{2} / 4 t\right)
$$

where $Y(t)$ is the Heaviside function. Suppose $K \subset \mathbf{R}^{n+1}$ is compact and contained in the half-space $\left\{(x, t): t<t_{0}\right\}$. Let $\chi$ denote the characteristic function of this half-space and let $\delta$ denote Lebesgue measure on the surface $t=t_{0}$. Notice that $\chi=\check{E} * \delta$. If $f=E * u$ where $u \in \tilde{E}_{K}^{\prime}\left(\mathbf{R}^{n+1}\right)$ then

$$
(H f, 1)=(u, \chi)=(u, \check{E} * \delta)=(E * u, \delta)=\int_{\mathbf{R}^{n}} f\left(x, t_{0}\right) d x .
$$

The next to last equality follows by Fubini's theorem if $u \in C_{0}^{\infty}\left(\mathbf{R}^{n+1}\right)$ and then for general $u \in \mathcal{E}_{K}^{\prime}\left(\mathbf{R}^{n+1}\right)$ by approximating and using the fact that $(E * u)\left(\cdot, t_{0}\right) \epsilon$ $L^{1}\left(\mathbf{R}^{n}\right)$. This provides an alternate definition of the $L^{\infty}$-capacity of $K$.

$$
L^{\infty}-\operatorname{Cap}_{E}(K)=\sup \left\{\left|\int f\left(x, t_{0}\right) d x\right|\right\}
$$


where the supremum is over potentials $f$ which are bounded in absolute value by 1 on $\mathbf{R}^{n+1}$. In regard to (3.4) see the remarks in Aronson [3].

Let $K \subset \mathbf{R}^{n+1}$ be compact. Denote by $F$ the generalized solution to the Dirichlet problem for $\mathbf{R}^{n+1} \sim K$ and $H$ with boundary values equal to one on $\partial K$. That is, $F$ is the balayage of the function 1 on $R^{n+1}$ relative to $K$. Let $\Omega_{K}$ denote the set of points in $\sim K$ which can be reached from $t=\infty$ by a curve with strictly decreasing $t$ coordinate.

Theorem 3.2. (a) The $L^{\infty}$-capacitary potential is unique in $\Omega_{K}$ and coincides with $F$ there.

(b) $\quad L^{\infty}-\operatorname{Cap}_{E}(K)=\sup \left\{\mu(1): \mu\right.$ is a positive measure on $K$ and $\left.\|E * \mu\|_{\infty} \leq 1\right\}$.

Remark. It can easily be shown that the capacitary potential is not unique on the whole unbounded component of $\mathbf{R}^{n+1} \sim K$. An example will suffice to illustrate what happens. Let $K=\{(x, t): 0 \leq t \leq 1,1 \leq|x| \leq 2\} \cup\{(x, t): 1 \leq t \leq 2$, $|x| \leq 2\}$. Let $g$ be any continuous function defined on $Q=\{(x, t): 0 \leq t \leq 1,|x| \leq 1\}$ such that $0 \leq g \leq 2, H g(x, t) \equiv 0$ in $Q^{\circ}$ and $g(x, 0) \equiv 0$. If $g$ is extended by 0 to all of $\mathbf{R}^{n+1}$, then $F-g$ is a capacitary potential for $K$.

Proof. Choose $t_{0}$ such that $K$ is contained in the half-space $\left\{(x, t): t<t_{0}\right\}$. Let $f$ be any capacitary potential for $K$. Since $|f(x, t)| \leq 1$ and $H f \equiv 0$ in $\mathbf{R}^{n+1} \sim K$, the definition of $F$ implies that $|f(x, t)| \leq F(x, t)$ in $\mathbf{R}^{n+1} \sim K$.

Hence, by (3.3),

$$
(H(F-f), 1)=\int\left[F\left(x, t_{0}\right)-f\left(x, t_{0}\right)\right] d x \geq 0 .
$$

Consequently, $(H F, 1) \geq(H f, 1)$, so $F$ is an $L^{\infty}$-capacitary potential for $K$. Then by (3.5) we have $F\left(x, t_{0}\right) \equiv f\left(x, t_{0}\right)$ for all $x$. Since $F(x, t) \geq f(x, t)$ for all $(x, t) \in \mathbf{R}^{n+1} \sim K$ we have $F(x, t)=f(x, t)$ for all $(x, t) \in \Omega_{K}$ by the maximum principle.

Since $F$ is an $L^{\infty}$-capacitary potential for $K$ and $H F$ is a positive measure, part (b) follows immediately.

The next example shows that the $L^{\infty}$-capacitary potential need not be unique. Let $\bar{\partial}^{2}$ denote the elliptic operator $\partial^{2} / \partial \bar{z}^{2}$ in C. The function $E(z)=\bar{z} / \pi z$ is a fundamental solution for $\bar{\partial}^{2}$. Using the homogeneity of $E$ we see that $L^{\infty}-\operatorname{Cap}_{E}(\lambda A)=L^{\infty}-\mathrm{Cap}_{E}(A)$ for all $\lambda>0$ and for all sets $A \subset \mathbf{R}^{2}$. Hence if $A$ is bounded we have $L^{\infty}-\operatorname{Cap}_{E}(A)=L^{\infty}-\operatorname{Cap}_{E}(\{0\})=\pi$. It is then obvious that if $K$ is compact and $z_{0} \in K$ the function $\pi E\left(z-z_{0}\right)$ is a capacitary potential for $K$. Consequently we have a high degree of nonuniqueness for the $L^{\infty}$-capacitary potential.

Remark. Suppose $P(D)$ has only constants for bounded entire solutions and has a fundamental solution $E$ such that each $P^{(\alpha)}(D) E$ vanishes at infinity. Then 
$L^{\infty}-\operatorname{Cap}_{E}(A) \leq L^{\infty}-\operatorname{Cap}_{P}(A) \leq 2 L^{\infty}-\operatorname{Cap}_{E}(A)$.

This can be seen as follows.

If $f \in L^{\infty}\left(\mathbf{R}^{n}\right),\|f\|_{\infty} \leq 1$, and $P f=0$ in $\mathbf{R}^{n} \sim K$, then $f-E * P f=c$.

Since $E * P f=E *(\phi P(D) f)=\sum\left(P^{(a)}(D) E\right) *\left(f D^{\alpha} \phi\right)$, where $\phi \in C_{0}^{\infty}\left(\mathbf{R}^{n}\right)$ with $\phi \equiv 1$ in a neighborhood of $K, E * P f$ vanishes at infinity, and hence $|c| \leq 1$. Therefore $E * P f \in L^{\infty}\left(\mathbf{R}^{n}\right)$ with $\|E * P f\|_{\infty} \leq 2$.

This remark applies to four of the five operators discussed above. In fact, one can easily show that $L^{\infty}-\operatorname{Cap}_{P}(A)=2 L^{\infty}-\operatorname{Cap}_{E}(A)$ if $P(D)$ is $d / d x, \Delta$, or $H$, and as noted above $L^{\infty}-\operatorname{Cap}_{\bar{\partial}}(A)=L^{\infty}-\operatorname{Cap}_{E}(A)$.

\section{BIBLIOGRAPHY}

1. D. Adams and J.C. Polking, The equivalence of two definitions of capacity (to appear).

2. L. Ahlfors, Bounded analytic functions, Duke Math. J. 14 (1947), 1-11. MR 9, 24.

3. D. G. Aronson, Removable singularities for linear parabolic equations, Arch. Rational Mech. Anal. 17 (1964), 79-84. MR 31 \#1470.

4. L. Carleson, Selected problems on exceptional sets, Van Nostrand Math. Studies, no. 13, Van Nostrand, Princeton, N.J., 1967. MR 37 \#1576.

5. E. B. Fabes and N. M. Riviere, Singular integrals with mixed homogeneity, Studia Math. 27 (1966), 19-38. MR $35 \# 683$.

6. B. Fuglede, Extremal length and functional completion, Acta Math. 98 (1957), $171-219$.

7. - Applications du théorème minimax à l'étude diverse capacités, C. R. Acad. Sci. Paris 266 (1968), 921-923.

8. P. R. Garabedian, Schwarz's lemma and the Szegö kernel function, Trans. Amer. Math. Soc. 67 (1949), 1-35. MR 11, 340.

9. R. Harvey and J.C. Polking, Removable singularities of linear partial differential equations, Acta. Math. 125 (1970), 39-56.

10. L. Hörmander, Linear partial differential operators, Die Grundlehren der math. Wissen schaften, Band 116, Academic Press, New York; Springer-Verlag, New York, 1963. MR 28 \#4221.

11. N. G. Meyers, A theory of capacities for potentials of functions in Lebesgue classes, Math. Scand. 26 (1970), 255-292.

12. J. C. Polking, Uniform approximation of nowhere dense sets by solutions of elliptic equations (in preparation).

13. Ju. G. Rešetnjak, The concept of capacity in the theory of functions with generalized derivatives, Sibirsk. Mat. ̌̌ 10 (1969), 1109-1138. (Russian)

DEPARTMENT OF MATHEMATICS, RICE UNIVERSITY, HOUSTON, TEXAS 77001 